\title{
Overexpression of activating transcription factor 3 exerts suppressive effects in HepG 2 cells
}

\author{
XIAOYAN LI ${ }^{1,2}$, SHENGBING ZANG $^{1}$, HAILI CHENG $^{1}$, JIASI LI $^{1}$ and AIMIN HUANG ${ }^{1}$ \\ ${ }^{1}$ Department of Pathology and Institute of Oncology, Fujian Medical University, Fuzhou, Fujian 350004; \\ ${ }^{2}$ Department of Pathology, Fujian Medical University Union Hospital, Fuzhou, Fujian 350001, P.R. China
}

Received March 2, 2018; Accepted October 9, 2018

DOI: $10.3892 / \mathrm{mmr} .2018 .9707$

\begin{abstract}
The present study observed and compared the biological behaviour of HepG2 cells prior and subsequent to the overexpression of activating transcription factor 3 (ATF3). Experiments investigating the cytological function by which ATF3 affects liver cancer cells were also performed. MTT, Transwell and flow cytometry assays were used to observe and detect the biological behaviour of HepG2 cells with and without lentivirus (LV)-ATF3-enhanced green fluorescent protein (EGFP) infection. The effects of ATF3 overexpression on cell proliferation, migration, apoptosis and cell cycle progression were evaluated. The LV-ATF3-EGFP overexpression vector was successfully constructed, and the HepG2 cells were successfully infected with the vector. Following ATF3 overexpression, cell proliferation was decreased, the rate of cell apoptosis was accelerated and cell cycle progression was slowed $(\mathrm{P}<0.05)$. There were no marked changes in cell migration $(\mathrm{P}>0.05)$, although there was a trend towards a gradual decrease. In conclusion, ATF3 exerted suppressive effects in HepG2 cells, potentially by inhibiting cancer cell growth, accelerating cell apoptosis, and blocking cell cycle progression. Intervention targeting ATF3 expression may represent a novel approach for the prevention and treatment of human liver cancer.
\end{abstract}

\section{Introduction}

Activating transcription factor 3 (ATF3) is a member of the ATF/cyclic adenosine 5'-monophosphate response element binding protein (CREB) subfamily. A role for the ATF/CREB family in controlling the progression of hepatocellular

Correspondence to: Professor Aimin Huang, Department of Pathology and Institute of Oncology, Fujian Medical University, 1 Xueyuan Road, Fuzhou, Fujian 350004, P.R. China

E-mail: draimin@163.com

Abbreviations: ATF3, activating transcription factor 3; HCC, hepatocellular carcinoma; LV, lentivirus

Key words: activating transcription factor 3, lentiviral vectors of overexpression, cytological mechanism carcinoma (HCC) has been suggested (1). ATF3 is an early stress response gene that is rapidly induced in cells following exposure to a wide range of stress stimuli (2). ATF3 may be rapidly induced in cells by a wide range of stress signals and may be activated by several important signal transduction pathways, including the mitogen activated protein kinase (MAPK) (3), c-myc avian myelocytomatosis viral oncogene (4), tumour protein p53 (p53) (5), and transforming growth factor- $\beta$ TGF- $\beta$ /Mothers against decapentaplegic (6) pathways, which are involved in cell proliferation, differentiation, transformation and apoptosis (7). The activation of ATF3 has been demonstrated to be a negative or positive regulator of these processes. Its precise effects remain controversial due to differences in the tumour types and cell lines examined. ATF3 has been indicated to promote (8) and inhibit (9) cellular proliferation and metastasis. In addition, it also has been suggested to exhibit pro- and anti-apoptotic effects, and to regulate cell cycle progression. To improve the understanding of how ATF3 acts in human HCC, the expression of ATF3 was evaluated in clinical samples of human HCC using quantitative polymerase chain reaction (qPCR), immunohistochemical (IHC) staining and a western blot analysis, and its expression was associated with the clinicopathological parameters of human HCC tissues in our previous study (10). It was identified that the expression of ATF3 was low in HCC tissues, and the protein level was decreased in patients with capsule invasion compared with those without. Therefore, it was inferred, based on the low expression of ATF3, that it may function as a tumour suppressor during human hepatocellular oncogenesis.

To improve the understanding of the cytological mechanisms by which ATF3 functions during the process of liver cancer formation, HepG2 cells were infected by an LV-ATF3-enhanced green fluorescent protein (EGFP) overexpression vector. Then, the biological behaviour of the cells with and without ATF3 overexpression was compared. The behaviours examined included cell proliferation, migration, apoptosis rate and cell cycle progression. The data from the present study may assist to clarify the association between ATF3 and human liver cancer.

\section{Materials and methods}

Cell culture. HepG2 cells were purchased from the Cellbank of Shanghai Institutes for Life Science, Chinese Academy of 
Sciences (Shanghai, China) and were maintained at $37^{\circ} \mathrm{C}$ in an atmosphere of $5 \% \mathrm{CO}_{2}$. Cell culture reagents were purchased from Thermo Fisher Scientific, Inc. (Waltham, MA, USA) unless otherwise indicated. The cells were grown in complete culture medium (high-glucose) consisting of Dulbecco's modified Eagle's medium (DMEM), supplemented with $10 \%$ heat-inactivated foetal bovine serum (FBS) and $1 \%$ penicillin/streptomycin.

Construction of lentiviral ATF3 overexpression vectors and cell transfection. Based on the ATF3 gene sequence in GenBank (NM_001674; https://www.ncbi.nlm.nih. gov/nuccore), primers that specifically matched ATF3 mRNA were designed (forward, GAGGATCCCCGGGTACCGGTC GCCACCATGATGCTTCAACACCCAGG; reverse, TCCTT GTAGTCCATACCGCTCTGCAATGTTCCTTCT; 587 bp). The lentiviral vector GV287-EGFP (Shanghai GeneChem Co., Ltd., Shanghai, China) was used to construct the ATF3 mRNA-expressing vector. The non-transfected group and mock vehicle group transfected with an empty vector without ATF3 mRNA were used as the negative control (NC). The GV287-EGFP vector was linearized by digestion with the restriction endonucleases $A g e \mathrm{I}$ and $\mathrm{BamHI}$, and the inserted sequences were ligated with an In-Fusion PCR Cloning kit (Clontech Laboratories, Inc., Mountainview, CA, USA) to produce the human ATF3 mRNA vectors (LV-ATF3-EGFP and LV-NC-EGFP). DNA sequencing was used to verify all inserted sequences. Using Lipofectamine ${ }^{\circledR} 2000$ (Invitrogen: Thermo Fisher Scientific, Inc.), 293T cells (Cellbank of Shanghai Institutes for Life Science, Chinese Academy of Sciences) were co-transfected with $20 \mu \mathrm{g}$ ATF3 mRNA vectors and the $15 \mu \mathrm{g}$ pHelper1.0 and $10 \mu \mathrm{g}$ pHelper2.0 plasmids (Addgene, Inc., Cambridge, MA, USA) for $48 \mathrm{~h}$ to generate lentiviruses. The viral titres were determined, and the lentiviral particles $2 \times 10^{9} \mathrm{TU} / \mathrm{ml}$ were used to infect HepG2 $2 \times 10^{5}$ cells at $37^{\circ} \mathrm{C}$ for $72 \mathrm{~h}$. Successfully transfected cells, which expressed EGFP, were selected and grown in cell culture for subsequent investigation.

Western blotting. HepG2 cells were lysed in ice-cold lysis buffer [150 mM NaCl, 100 mM Tris (pH 8.0), 1\% Tween-20, 1 mM EDTA, $1 \mathrm{mM}$ phenylmethylsulfonyl fluoride, $10 \mu \mathrm{g} / \mathrm{ml}$ aprotinin, $10 \mu \mathrm{g} / \mathrm{ml}$ trypsin, and $10 \mu \mathrm{g} / \mathrm{ml}$ leupeptin]. The lysate was left on ice for $20 \mathrm{~min}$ and then centrifuged at $4^{\circ} \mathrm{C}, 1,200 \mathrm{x} \mathrm{g}$ for $10 \mathrm{~min}$. The clarified supernatant was collected, and the protein concentration was measured by using a Biotek protein assay kit (Elx800; BioTek Instruments, Winooski, VT, USA). Protein lysate $(60 \mu \mathrm{g}$ per well), was separated by $12 \%$ SDS-PAGE and transferred to polyvinylidene difluoride membranes. Membranes were incubated with 5\% non-fat milk for $1 \mathrm{~h}$ at room temperature and then with mouse monoclonal anti-human ATF3 antibody (1:100 dilution, ab58668; Abcam, Cambridge, MA, USA) at $4^{\circ} \mathrm{C}$ overnight. The membranes were washed and stained with a horseradish-peroxidase-conjugated secondary antibody (at 1:1,000 dilution, A0216, Beyotime Institute of Biotechnology, Shanghai, China). Proteins were visualized using Enhanced Chemiluminescence Plus system (Beyotime Institute of Biotechnology) and exposed to autoradiography film (Kodak, Rochester, NY, USA). Blots with mouse monoclonal $\beta$-Actin antibody (at 1:1,000 dilution, AA128; Beyotime Institute of Biotechnology) were similarly generated to ensure that equal amounts of protein were loaded in the wells.

Cell proliferation. HepG2 cells were divided into three groups: An overexpression group (OE); a mock vehicle-transfected negative control group (NC); and a non-transfection group (control).

Cell proliferation was measured using an MTT colorimetric assay from Beijing Dingguo Changsheng Biotechnology Co. Ltd. (Beijing, China). All the cells were seeded in 96-well plates and cultured with complete culture medium. Cell proliferation was measured on days 1-5 at $490 \mathrm{~nm}$ in an ELIASA plate reader (Elx800; BioTek Instruments) following the addition of dimethyl sulfoxide to each well. The cell proliferation rate was calculated relative to the optical density at $490 \mathrm{~nm}$ of day 1 .

Cell migration. Transwell migration assays were performed in 24-well dishes with cell culture plate inserts (Corning Incorporated, Corning, NY, USA). The upper chamber had a $3 \mu \mathrm{m}$ pore size and was coated with a polycarbonate membrane, with $100 \mu \mathrm{l}$ serum-free medium/well, and the plates were incubated at $37^{\circ} \mathrm{C}$ for $1-2 \mathrm{~h}$. The lower chamber consisted of $600 \mu 1$ DMEM medium supplemented with $10 \%$ FBS. Actively growing cells $\left(1 \times 10^{5}\right.$ cells) were diluted in $100 \mu 1$ serum-free medium supplemented with $0.1 \%$ bovine serum albumin (Absin Biochemical Company, Shanghai, China), added to the upper chamber and incubated at $37^{\circ} \mathrm{C}$ for $16 \mathrm{~h}$. Following incubation, the medium from the upper chamber was removed, and the cells on the upper surface of the membrane were rinsed and removed with a cotton swab. The upper chambers were stained at $37^{\circ} \mathrm{C}$ for $20 \mathrm{~min}$ with Giesma solution (Sigma-Aldrich; MercK KGaA, Darmstadt, Germany). Images of the migrated cells were captured by an inverted fluorescence microscope (magnification, x100, Olympus Corporation, Tokyo, Japan), and their optical densities at $570 \mathrm{~nm}$ were measured by ELIASA microplate reader (Elx800; Bio Tek Instruments).

Cell apoptosis. HepG2 cells were collected following infection with the lentiviruses for 5 days; then, the cells were washed twice with cold PBS and binding buffer. The collected cells were resuspended in staining buffer and then added into Annexin V-allophycocyanin (APC) staining solution and incubated for 10-15 min at room temperature in the dark. Flow cytometric analysis was performed using a FACSCalibur instrument (BD Biosciences, Franklin Lakes, NY, USA). The results were analysed by FlowJo7.6 software (FlowJo LLC, Ashland, OR, USA).

Cell cycle progress. HepG2 cells (third to fourth generation) were collected following infection with the lentivirus; then, the collected cells were washed and resuspended with cold PBS. Subsequent to fixation with $70 \%$ ethyl alcohol at $4^{\circ} \mathrm{C}$ overnight, the cell suspension was added into $50 \mu \mathrm{g} / \mathrm{ml}$ propidium iodide staining solution and incubated for $30 \mathrm{~min}$ at $37^{\circ} \mathrm{C}$ in the dark. Flow cytometric analysis was performed using a FACSCalibur instrument, and the results were analysed using FlowJo7.6 software (FlowJo LLC).

Statistical analysis. The statistical package SPSS 19.0 was used to perform the statistical analyses. $\mathrm{P}<0.05$ was considered to indicate a statistically significant difference. 


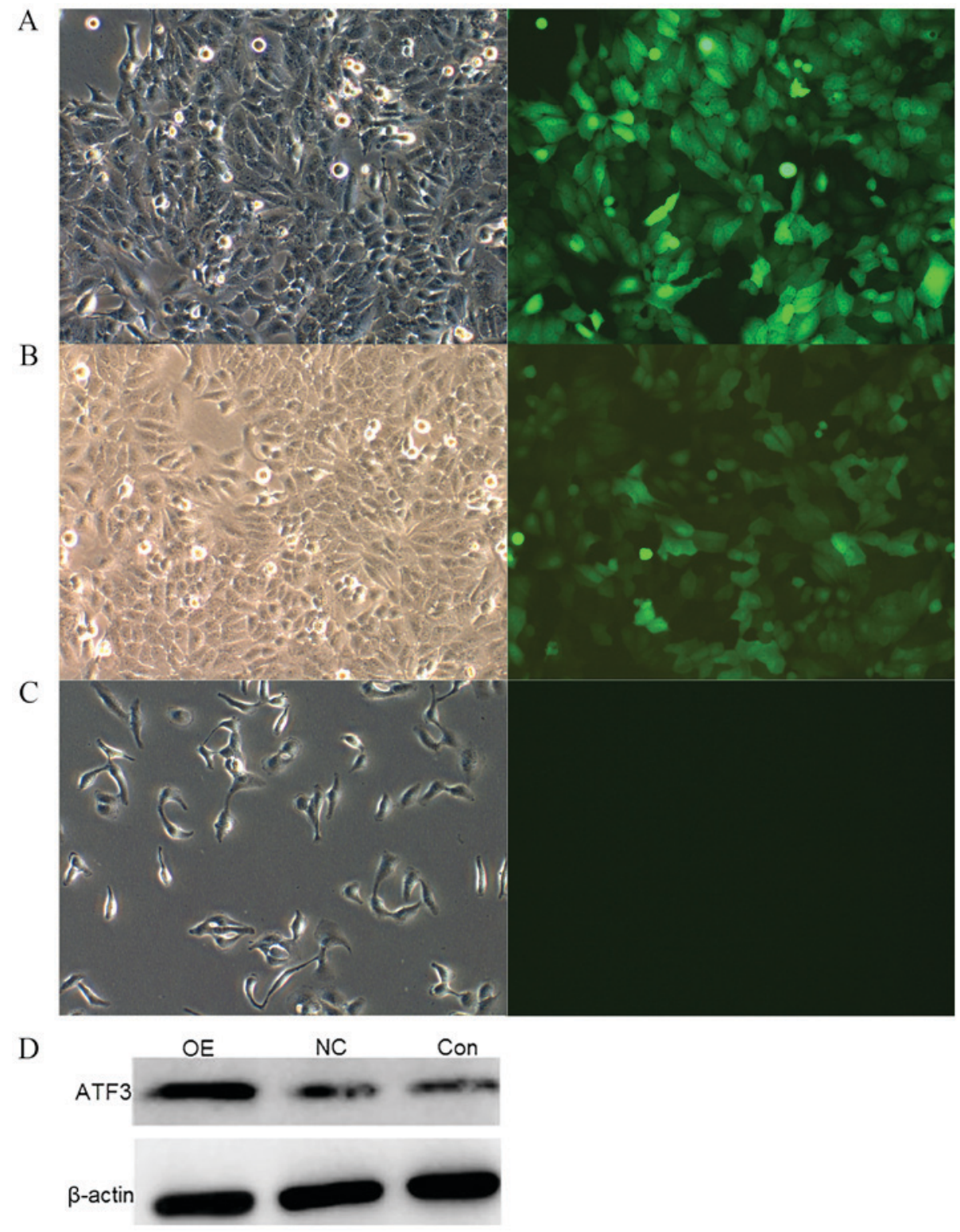

Figure 1. Overexpression of ATF3 in HepG2 cells is determined by fluorescence microscope and western blot. A total of $>90 \%$ cells with the green fluorescence were identified in the (A) OE and (B) NC groups. (C) No signal was measured in the control group, as determined by fluorescence microscope. Magnification, x100. (D) ATF3 protein level in HepG2 cells was determined by western blot. It showed an increase in OE group compared with the NC and control groups was observed visually. ATF3, activating transcription factor 3; OE, overexpression; NC, negative control.

The optical density (OD) 490 value, OD570/OD490 value, apoptotic rate and cell percentages were representative of the MTT, Transwell, apoptosis and cell cycle results, respectively. All results presented as mean \pm standard deviation. Tumour-associated variables in the Transwell and apoptosis results were examined using a one-way analysis of variance (ANOVA) to compare the differences among groups, and followed by a Least Significant Difference test between groups. For the variables associated with the MTT and cell cycle assays, statistical significance was determined according to an ANOVA with a randomized block design, followed by a Least Significant Difference test.

\section{Results}

To investigate the functional significance of ATF3 expression in HepG2 cells, HepG2 cells were transfected with lentiviral vectors expressing ATF3 mRNA. The mRNA expression was associated with increased ATF3 protein expression, as demonstrated in Fig. 1. The MTT assay indicated that the overexpression of ATF3 resulted in HepG2 growth inhibition (Fig. 2). However, there were no marked differences in the cell migration detected by the Transwell assay, as presented in Fig. 3. Figs. 4 and 5 indicated that the rate of HepG2 apoptosis was accelerated and that the cells underwent cycle arrest following ATF3 overexpression, respectively, as determined by flow cytometric analyses.

ATF3 is successfully overexpressed in HepG2 cells. To examine the biological activity of ATF3, ATF3 was cloned into the lentiviral vector GV287-EGFP. Stable pools of HepG2 cells containing full-length ATF3 were generated. The overexpression of ATF3 was confirmed in these cells by fluorescence microscope and western blot analysis. Relative to the NC and 

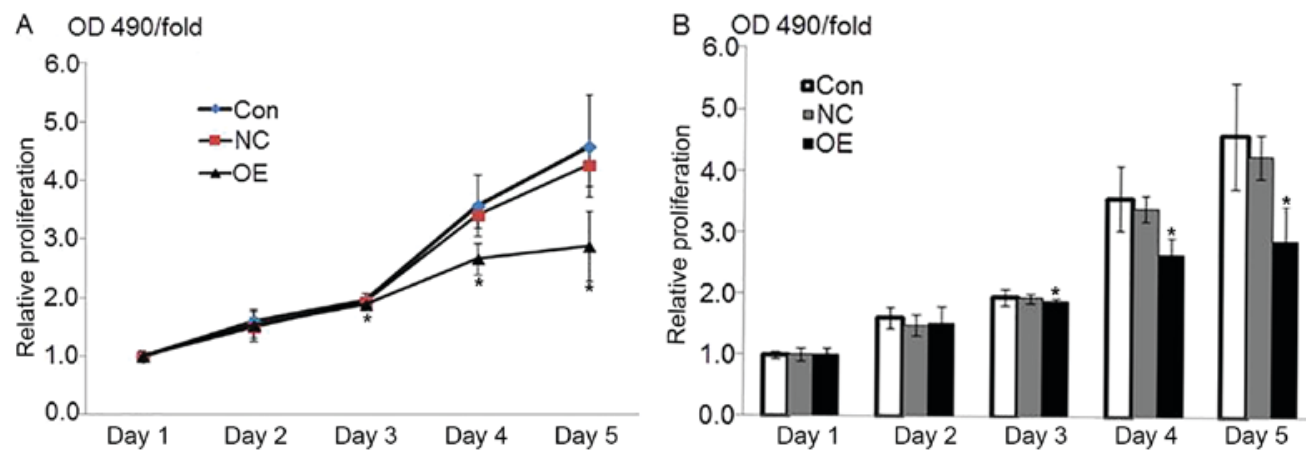

Figure 2. HepG2 proliferation ability following the overexpression of ATF3 is detected by MTT assay. (A) Growth curves of HepG2 cells in OE, NC and control groups. (B) Composite histograms of OD490/fold values in $\mathrm{OE}, \mathrm{NC}$ and control groups. The OD490/fold value in OE group was significantly decreased compared with the $\mathrm{NC}$ and control groups from the third day following infection, while there was no significance between the NC and control groups $(\mathrm{P}=0.637) .{ }^{*} \mathrm{P}<0.05$ vs. control and $\mathrm{NC}$ groups. Data are presented as the mean \pm standard deviation. ATF3, activating transcription factor 3; OE, overexpression; NC, negative control; Con, control.
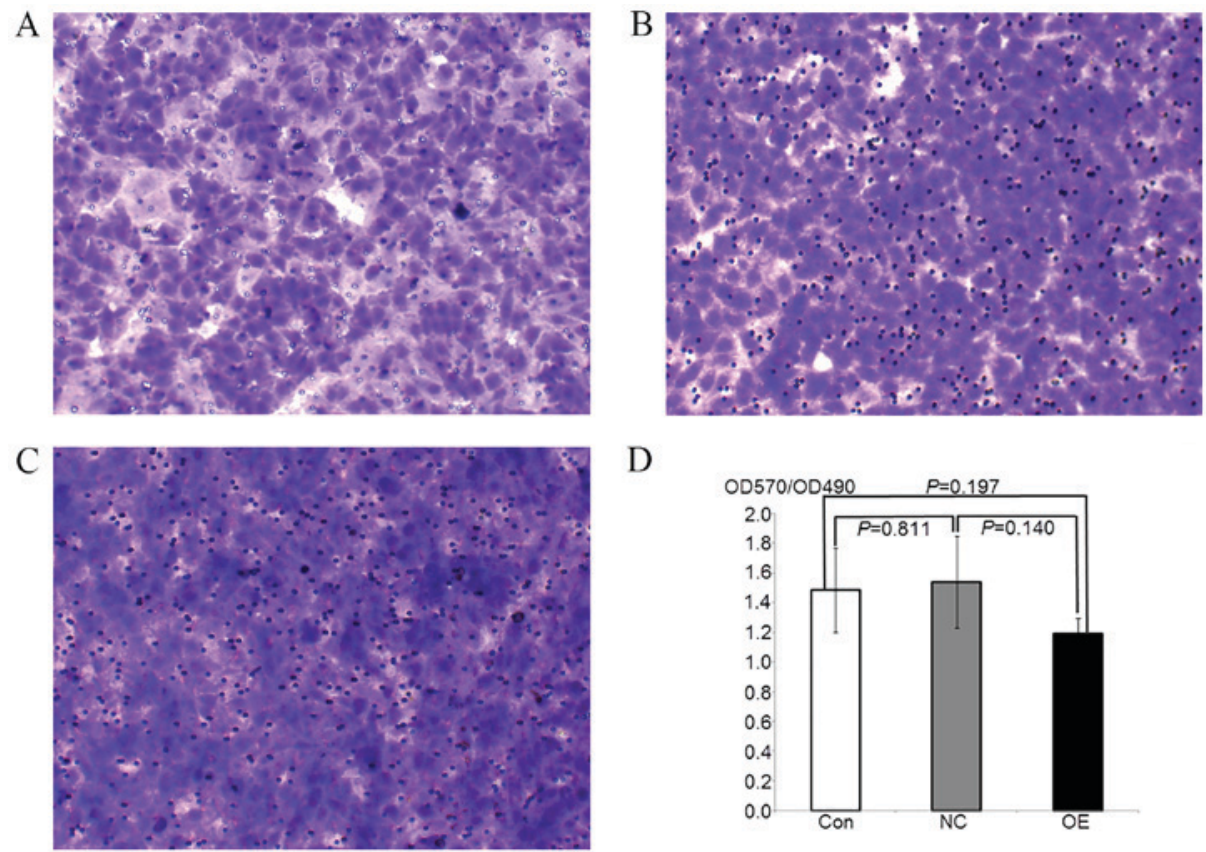

D

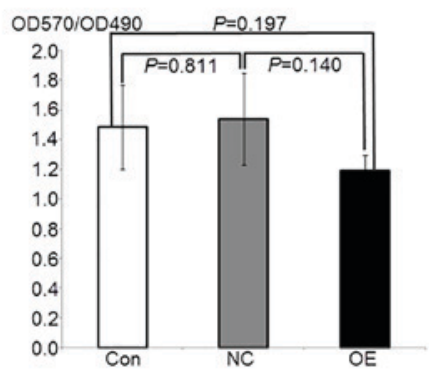

Figure 3. HepG2 transfer ability following overexpression of ATF3 is detected by Transwell assay. (A-C) Microscope images of Giesma-stained HepG2 transfer cells in (A) OE, (B) NC and (C) control groups. Magnification, x100. (D) Histograms of OD570/OD490 values in OE, NC and control groups. Data are presented as the mean \pm standard deviation. The transfer difference at the $\mathrm{OE}, \mathrm{NC}$ and control groups was not marked, meanwhile no significance between groups was observed $(\mathrm{P}>0.05)$. ATF3, activating transcription factor 3; OE, overexpression; NC, negative control; Con, control; OD, optical density.

control groups, the OE group exhibited a marked increase in ATF3 protein expression (Fig. 1).

Overexpression of ATF3 inhibits the growth of HepG2 cells. The optical density at $490 \mathrm{~nm}$ on the first day was considered the reference coefficient. The percent viability was calculated relative to this value as the fold-change (OD490/fold) from the second day to the fifth day. The MTT-OD490 values were representative of cell growth activity. The results indicated that the value in the OE group was significantly decreased compared with that in the $\mathrm{NC}$ and control groups beginning from the third day following lentiviral vector infection $(\mathrm{P}<0.05)$, whereas no significant differences between the NC and control groups were observed ( $\mathrm{P}=0.637$; Fig. 2). These data indicated that the overexpression of ATF3 may inhibit the growth of HepG2 cells.
Overexpression of ATF3 does not affect the migration of $H e p G 2$ cells. To examine the effect of cell proliferation on the number of cells that migrated across the Transwell membrane, the optical density at $570 \mathrm{~nm}$ was calculated relative to the corresponding MTT-OD490 values. The OD570/OD490 values were representative of the numbers of cells that migrated through the chamber membrane. The results indicated no significant differences in the number of cells that migrated in the $\mathrm{OE}, \mathrm{NC}$ and control groups, and there were no significant differences between the various groups $(\mathrm{P}>0.05$; Fig. 3). These data indicated that the overexpression of ATF3 had no significant effects on the migration of HepG2 cells.

Overexpression of ATF3 increases the apoptotic activity in Hep 62 cells. The APC fluorescence intensity was examined by flow cytometry in the FL4 channel, and the fluorescence 

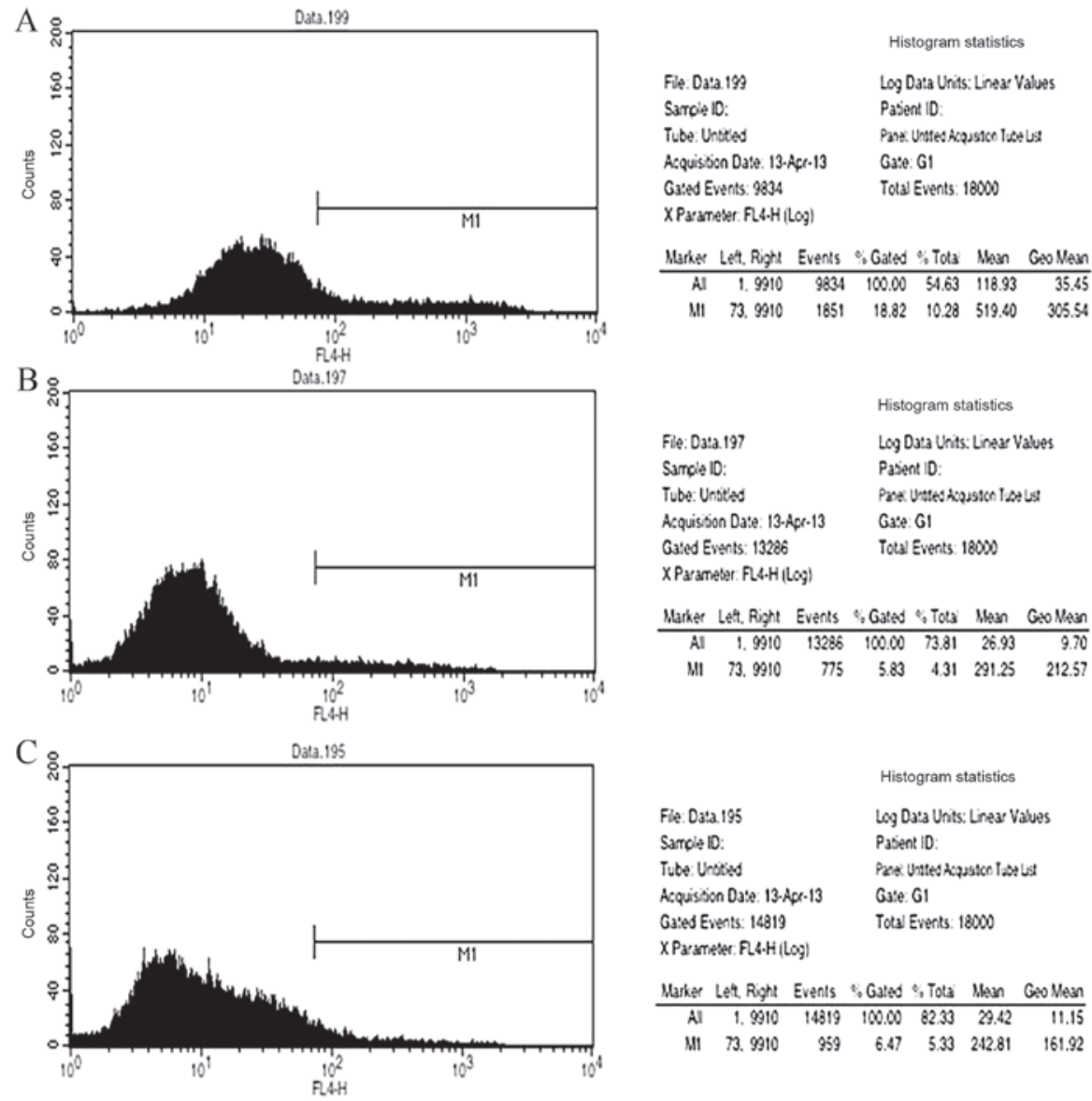

D Apoptosis (\%) $P=0.000^{*}$

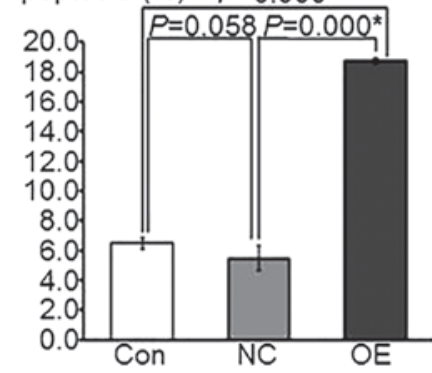

Figure 4. HepG2 apoptotic rate following the overexpression of ATF3 was detected by Annexin V-allophycocyanin flow cytometry. (A-C) Peak pattern diagrams of the (A) OE, (B) NC and (C) control groups. The percentage of cells in the 'M1' region is representative of the apoptotic rate. The value in OE group was increased compared with the $\mathrm{NC}$ and control groups. (D) Peak pattern histograms of apoptotic rate in OE, NC and control groups. The HepG2 apoptotic rate in the $\mathrm{OE}$ group was significantly increased compared with the $\mathrm{NC}$ and control groups ( $\mathrm{P}<0.05)$, while no significance between the $\mathrm{NC}$ and control groups was observed $(\mathrm{P}=0.058)$. Data are presented as the mean \pm standard deviation. ATF3, activating transcription factor 3; OE, overexpression; NC, negative control; Con, control.

intensity of EGFP, which was used as a reporter gene, was measured in the FL1 channel. The percentage of apoptotic cells, which was representative of the cell apoptotic rate, was calculated by comparing these 2 fluorescence intensities. The results demonstrated that the apoptotic rate in the $\mathrm{OE}$ group was significantly increased compared with that in the $\mathrm{NC}$ and control groups $(\mathrm{P}<0.05)$, whereas there was no significant difference between the $\mathrm{NC}$ and control groups $(\mathrm{P}=0.058$; Fig. 4). These data suggest that the overexpression of ATF3 may increase the apoptosis of $\mathrm{HepG} 2$ cells.

Overexpression of ATF3 decreases cell cycle progression in HepG2 cells. The numbers of cells in each phase of the cell cycle were detected by flow cytometry using a FACSCalibur instrument. The cell proportions in various phases of the cell cycle were calculated to demonstrate the specific cell growth states. The results indicated that the proportion of cells in the G0/G1 phase in the OE group was significantly increased compared with that in the $\mathrm{NC}$ and control groups $(\mathrm{P}<0.05)$, whereas the proportions of cells in the $\mathrm{S}$ and $\mathrm{G} 2 / \mathrm{M}$ phases were not significantly different compared with the other groups $(\mathrm{P}>0.05)$. There was no significant difference between the $\mathrm{NC}$ and control groups in any of the phases of the cell cycle $(\mathrm{P}=0.183)$. The sum of the $\mathrm{S}$ and $\mathrm{G} 2 / \mathrm{M}$ cell proportions was representative of cell proliferation. The results were the same as those of the MTT assay, which suggested that the sum of the $\mathrm{S}$ and $\mathrm{G} 2 / \mathrm{M}$ cell proportions in the OE group was significantly decreased compared with that in the NC and control 


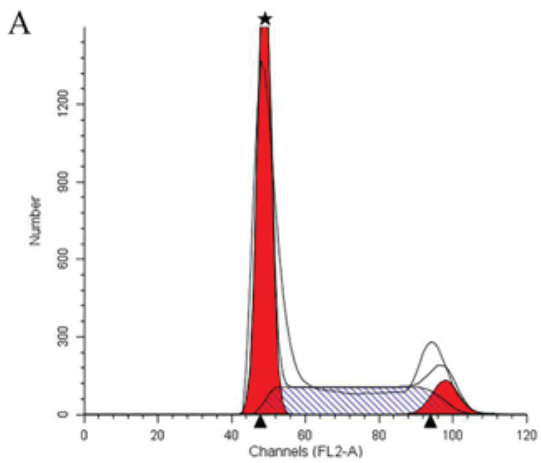

Ploidy mode: first cycle is diploid

Diploid: $100.00 \%$

Dip G1: $56.41 \%$ at 49.03

Dip G2: $8.11 \%$ at 98.06

Dip S: $35.47 \%$ G2/G1:2.00

\% CV: 3.68

Total S-Phase: $35.47 \%$

TotalB.A.D.: $0.00 \%$ no debris no aggs
B

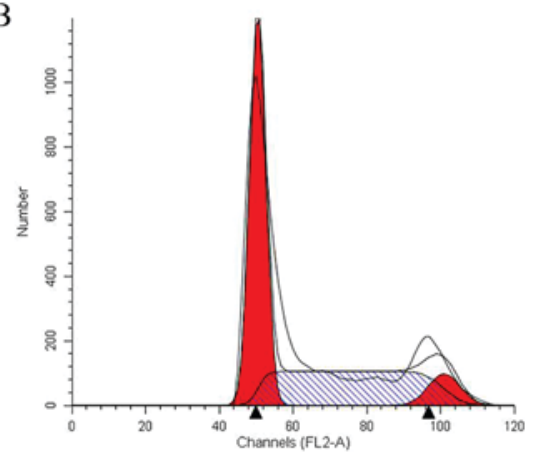

Ploidy mode: first cycle is diploid

Diploid: $100.00 \%$

Dip G1: $51.18 \%$ at 50.58

Dip G2: $8.15 \%$ at 101.16

Dip S: $40.67 \%$ G2/G1:2.00

\% CV: 4.28

Total S-Phase: $40.67 \%$

TotalB.A.D.: $0.00 \%$ no debris no aggs

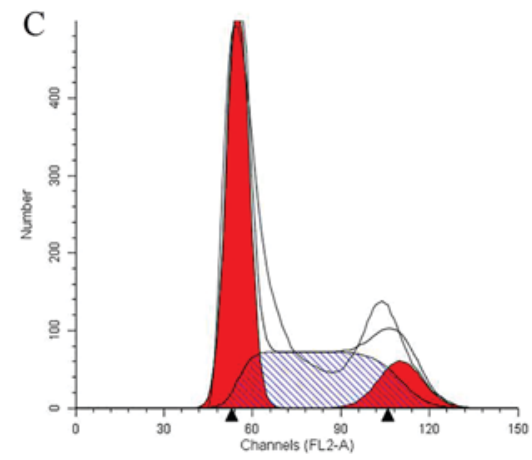

Ploidy mode: first cycle is diploid

Diploid: $100.00 \%$

Dip G1: $49.81 \%$ at 55.17

Dip G2: $11.18 \%$ at 110.35

Dip S: $39.00 \%$ G2/G1:2.00

\% CV: 6.78

Total S-Phase: $39.00 \%$

TotalB.A.D.: $0.00 \%$ no debris no aggs
D

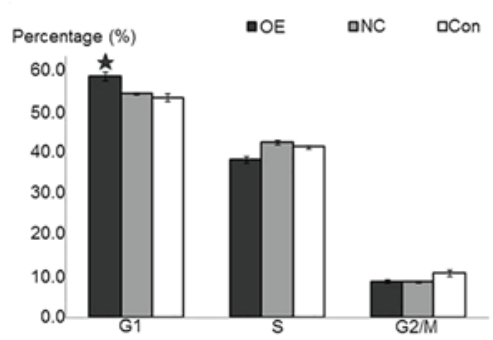

E

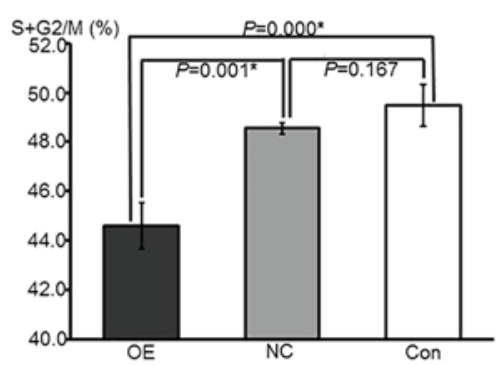

Figure 5. HepG2 cell cycle progression following overexpression of ATF3 was detected by propidium iodide staining. (A-C) Flow cytometry cycle diagram of (A) $\mathrm{OE},(\mathrm{B}) \mathrm{NC}$ and (C) Con groups. DipG1 was representative of the proportion of cells in the G0/G1 phase; DipG2 was representative of the proportion of cells in the G2/M phase; and DipS was representative of the proportion of cells in the S phase. (D) Composite histograms of the cell proportion in G0/G1, S and G2/M phases among the OE, NC and Control groups. Data are presented as the mean \pm standard deviation. The G0/G1 proportion in OE group was significantly increased compared with the $\mathrm{NC}$ and control groups, while the $\mathrm{S}$ and $\mathrm{G} 2 / \mathrm{M}$ proportion were not significance compared with other groups. "P<0.05 vs. NC and control groups. (E) Histograms of the $\mathrm{S}+\mathrm{G} 2 / \mathrm{M}$ phase cell proportions in the $\mathrm{OE}, \mathrm{NC}$ and control groups. Data are presented as the mean \pm standard deviation. The proportion sum in OE group was significantly decreased compared with the NC and control groups, while no significance between the NC and control groups was observed. ${ }^{*} \mathrm{P}<0.05$ vs. $\mathrm{NC}$ and control groups. ATF3, activating transcription factor 3; OE, overexpression; NC, negative control; Con, control.

groups $(\mathrm{P}<0.05)$, whereas there was no significant difference between the $\mathrm{NC}$ and control groups $(\mathrm{P}=0.167$; Fig. 5). These data indicated that the overexpression of ATF3 inhibited cell proliferation by inducing cell cycle arrest at the G0/G1 phase.

\section{Discussion}

Lentiviral vectors are now the most commonly used tool in genetic intervention experiments. GV287, pHelper1.0 and pHelper2.0 plasmids were used as an integrated overexpression system for the target gene ATF3 in the present study. HepG2 cells were successfully infected with the recombinant plasmid generated in the Institute of Oncology, with a marked green fluorescent signal and a high level of ATF3 protein expression.

Following the overexpression of ATF3 in HepG2 cells, proliferation was markedly inhibited from the third day, as indicated by the MTT assay. From the third day until the fifth day following infection, the proliferation of the HepG2 cells in the OE group was significantly decreased compared with that in the NC and control groups. Similar to the results of the MTT assay, the sum of the cells in the $\mathrm{S}$ and $\mathrm{G} 2 / \mathrm{M}$ phases of the cell cycle in the OE group was also markedly decreased compared with that in the other groups, as detected by flow cytometry. In addition, the proportion of cells in the G0/G1 phase in the OE group was significantly increased compared with that in the $\mathrm{NC}$ and control groups. These consistent results demonstrated that cell cycle progression in HepG2 cells may be blocked in the mitosis stage by the overexpression of the ATF3 protein. Ultimately, cell viability was decreased in the tumour cells with high ATF3 levels.

Additionally, following the overexpression of ATF3 in HepG2 cells, the apoptotic rate in the OE group was markedly increased compared with that in the $\mathrm{NC}$ and control groups, as detected by flow cytometry. The rates of tumour cell apoptosis were accelerated by the high level of ATF3 protein. However, HepG2 migration was not markedly different among the three groups as a whole, as indicated by the Transwell assay. Nevertheless, the OD570/OD490 value, which represents cell mobility, was decreased in the OE group compared with the $\mathrm{NC}$ and control groups. Therefore, there was a tendency for the HepG2 cells with ATF3 overexpression to exhibit decreased cell migration. These results were consistent with our previous 
study (10), which demonstrated that capsule invasion was weaker in liver cancer tissues with a high level of ATF3 protein.

Combined with the data from our previous study (10), our results indicate that the ATF3 level is low in human HCC tissues, and there was a decreased protein level in patients with capsule invasion. All these results indicate that ATF3 may serve a role as a tumour suppressor during human hepatocellular oncogenesis.

The present study provides mechanistic insights into this phenomenon of ATF3 as a tumour suppressor. The changes in the biological behaviour of HepG2 cells with and without ATF3 overexpression were explored, and it was observed that the cells overexpressing ATF3 exhibited slower viability, accelerated apoptosis and cell cycle arrest. These data provide insight into how ATF3 affects liver cancer cells.

According to previous studies, ATF3 is primarily activated by 2 important signal transduction pathways, c-Jun N-terminal kinase/stress-activated protein kinase (JNK/SAPK) and P38/MAPK (11). Certain studies have identified that persistent activation of the JNK/SAPK signalling pathway is closely associated with cell apoptosis (12), and the P38/MAPK pathway is crucial for promoting apoptosis (13). ATF3 suppresses oncogenic networks, induces cell death and inhibits malignant dissemination in a number of cancer types (14), including glioblastoma and colon, prostate, bladder and lung cancer (9,15-18). In a study by Wang et al (19), the expression of the NOXA gene, which is a key mediator of apoptosis, may be enhanced by ATF3. All these data indicate that ATF3 expression and tumour cell death are closely associated. Certain studies hypothesize that this association is likely to be context-dependent $(20,21)$.

In addition, several studies have revealed that ATF3 may induce cell cycle arrest to decrease tumour formation. A study by Feng et al (22) explored whether ATF3 may regulate lung small cancer cells proliferation through regulation of the cyclin D1-associated pathway. In an additional study, the ATF3 protein was demonstrated to slow cell progression from the G1 to $S$ phase, and to moderately suppress cell growth (23). A study by James et al (24) demonstrated decreased cyclin-dependent kinase activity and hypophosphorylation of pocket proteins in response to ATF3 upregulation in mature chondrocytes, which would have resulted in cell cycle exit and increased activity of runt-related transcription factor 2 , thereby terminating chondrocyte differentiation. Additionally, there is certain evidence indicating that ATF3 may suppress cell migration and invasion $(9,25)$. These results agree with the data from the present study. At present, our studies on HepG2 cells have verified certain basic results regarding the association between ATF3 and tumour formation and have illustrated that a high level of ATF3 may inhibit liver cancer development.

However, ATF3 is unlikely to be solely responsible for the development of liver cancer. The context-dependent role that ATF3 serves in tumours is due to the intricate networks formed with other genes and proteins. Future studies will focus on the protein-protein interactions of ATF3 to investigate how ATF3 and its critical partners function in liver cancer oncogenesis. Whether an intervention targeting ATF3 may be used as a novel strategy for liver cancer prevention and treatment is an interesting question and remains to be resolved.

\section{Acknowledgements}

Not applicable.

\section{Funding}

The present study was supported by grants from Fujian Provincial Education Office (grant no. 2013B009) and The Special Fund of Fujian Provincial Finance Department of China (grant no. 2014-1262).

\section{Availability of data and materials}

The data and materials are available from the first author and corresponding author on reasonable request.

\section{Authors' contributions}

XL made substantial contributions to analyzing data and the writing of the manuscript. SZ performed the construction of lentiviral ATF3 overexpression vectors and cell transfection. $\mathrm{HC}$ and JL performed the examination of biological behaviour of HepG2 cells. AH made substantial contributions to the design of the present study and quality control. All the authors have approved the final version of the manuscript.

\section{Ethics approval and consent to participate}

Not applicable.

\section{Patient consent for publication}

Not applicable.

\section{Competing interests}

The authors declare that they have no competing interests.

\section{References}

1. Brunacci C, Piobbico D, Bartoli D, Castelli M, Pieroni S, Bellet MM, Viola-Magni M, Della Fazia MA and Servillo G: Identification and characterization of a novel peptide interacting with cAMP-responsive elements binding and cAMP-responsive elements modulator in mouse liver. Liver Int 30: 388-395, 2010.

2. Gilchrist M, Henderson WR Jr, Morotti A, Johnson CD, Nachman A, Schmitz F, Smith KD and Aderem A: A key role for ATF3 in regulating mast cell survival and mediator release. Blood 115: 4734-4741, 2010.

3. Du Z and Jiang B: The research progress of ATF3 and tumor. J Gannan Univ Med 30: 163-166, 2010.

4. Tamura K, Hua B, Adachi S, Guney I, Kawauchi J, Morioka M, Tamamori-Adachi M, Tanaka Y, Nakabeppu Y, Sunamori M, et al: Stress response gene ATF3 is a target of c-myc in serum-induced cell proliferation. EMBO J 24: 2590-2601, 2005.

5. Taketani K, Kawauchi J, Tanaka-Okamoto M, Ishizaki H, Tanaka Y, Sakai T, Miyoshi J, Maehara Y and Kitajima S: Key role of ATF3 in p53-dependent DR5 induction upon DNA damage of human colon cancer cells. Oncogene 31: 2210-2221, 2012.

6. Yin X, Wolford CC, Chang YS, McConoughey SJ, Ramsey SA, Aderem A and Hai T: ATF3, an adaptive-response gene, enhances TGF-\{beta\} signaling and cancer-initiating cell features in breast cancer cells. J Cell Sci 123: 3558-3565, 2010.

7. Ma S, Pang C, Song L, Guo F and Sun H: Activating transcription factor 3 is overexpressed in human glioma and its knockdown in glioblastoma cells causes growth inhibition both in vitro and in vivo. Int J Mol Med 35: 1561-1573, 2015. 
8. TanakaY,NakamuraA,MoriokaMS,InoueS,Tamamori-AdachiM, Yamada K, Taketani K, Kawauchi J, Tanaka-Okamoto M, Miyoshi J, et al: Systems analysis of ATF3 in stress response and cancer reveals opposing effects on pro-apoptotic genes in p53 pathway. PLoS One 6: e26848, 2011.

9. HacklC,Lang SA, Moser C, Mori A, Fichtner-Feigl S, Hellerbrand C, Dietmeier W, Schlitt HJ, Geissler EK and Stoeltzing O: Activating transcription factor-3 (ATF3) functions as a tumor suppressor in colon cancer and is up-regulated upon heat-shock protein 90 (Hsp90) inhibition. BMC Cancer 10: 668, 2010.

10. Xiaoyan L, Shengbing Z, Yu Z, Lin Z, Chengjie L, Jingfeng L and Aimin $\mathrm{H}$ : Low expression of activating transcription factor 3 in human hepatocellular carcinoma and its clinicopathological significance. Int J Mol Med 210: 477-481, 2014.

11. Maciag AE, Nandurdikar RS, Hong SY, Chakrapani H, Diwan B, Morris NL, Shami PJ, Shiao YH, Anderson LM, Keefer LK and Saavedra JE: Activation of the c-Jun N-terminal kinase/activating transcription factor 3 (ATF3) pathway characterizes effective arylated diazeniumdiolate-based nitric oxide-releasing anticancer prodrugs. J Med Chem 54: 7751-7758, 2011.

12. Wang J, Sun F, Sun W, Shi H, Yong Y,Liu S and Liu L: Busuishengxue ranules mediate their effects upon non-severe aplastic anemia via mitogen-activated protein kinase/extracellular signal-regulated kinase pathway. J Tradit Chin Med 34: 23-29, 2014

13. Slattery ML, Lundgreen A and Wolff RK: Dietary influence on MAPK-signaling pathways and risk of colon and rectal cancer. Nutr Cancer 65: 729-738, 2013.

14. Wei S, Wang H, Lu C, Malmut S, Zhang J, Ren S, Yu G, Wang W, Tang DD and Yan C: The activating transcription factor 3 protein suppresses the oncogenic function of mutant $\mathrm{p} 53$ proteins. J Biol Chem 289: 8947-8959, 2014

15. Gargiulo G, Cesaroni M, Serresi M, de Vries N, Hulsman D, Bruggeman SW, Lancini $\mathrm{C}$ and van Lohuizen $\mathrm{M}$ : In vivo RNA screen for BMI1 targets identifies TGF- $3 /$ BMP-ER stress pathways as key regulators of neural- and malignant glioma-stem cell homeostasis. Cancer Cell 23: 660-676, 2013.

16. Huang X, Li X and Guo B: KLF6 induces apoptosis in prostate cancer cells through up-regulation of ATF3. J Biol Chem 283: 29795-29801, 2008

17. Yuan X, Yu L, Li J, Xie G, Rong T, Zhang L, Chen J, Meng Q, Irving AT, Wang D, et al: ATF3 suppresses metastasis of bladder cancer by regulating gelsolin-mediated remodeling of the actin cytoskeleton. Cancer Res 73: 3625-3637, 2013.
18. Jan YH, Tsai HY, Yang CJ, Huang MS, Yang YF, Lai TC, Lee CH, Jeng YM, Huang CY, Su JL, et al: Adenylate kinase-4 is a marker of poor clinical outcomes that promotes metastasis of lung cancer by downregulating the transcription factor ATF3. Cancer Res 72: 5119-5129, 2012.

19. Wang Q, Mora-Jensen H, Weniger MA, Perez-Galan P, Wolford C, Hai T, Ron D, Chen W, Trenkle W, Wiestner A and Ye Y: ERAD inhibitors integrate ER stress with an epigenetic mechanism to activate $\mathrm{BH} 3$-only protein NOXA in cancer cells. Proc Natl Acad Sci USA 106: 2200-2205, 2009.

20. Lu D, Wolfgang CD and Hai T: Activating transcription factor ATF3, a stress inducible gene, suppresses Ras stimulated tumorigenesis. J Biol Chem 281: 10473-10481, 2006.

21. Wang G, Lunardi A, Zhang J, Chen Z, Ala U, Webster KA, Tay Y, Gonzalez-Billalabeitia E, Egia A, Shaffer DR, et al: Zbtb7a suppresses prostate cancer through repression of a Sox9-dependent pathway for cellular senescence bypass and tumor invasion. Nat Genet 45: 739-746, 2013.

22. Feng J, Sun Q, Wu T, Lu J, Qu L, Sun Y, Tian L, Zhang B, Li D and Liu M: Upregulation of ATF-3 is correlated with prognosis and proliferation of laryngeal cancer by regulating Cyclin D1 expression. Int J Clin Exp Pathol 6: 2064-2070, 2013.

23. Fan F, Jin S, Amundson SA, Tong T, Fan W, Zhao H, Zhu X, Mazzacurati L, Li X, Petrik KL, et al: ATF3 induction following DNA damages regulated by distinct signaling pathways and over expression of ATF3 protein suppresses cells growth. Oncogene 21: 7488-7496, 2002.

24. James CG, Woods A, Underhill TM and Beier F: The transcription factor ATF3 is up-regulated during chondrocyte differentiation and represses cyclin D1 and A gene transcription. BMC Mol Biol 7: 30, 2006.

25. Zigler M, Villares GJ, Dobroff AS, Wang H, Huang L, Braeuer RR, Kamiya T, Melnikova VO, Song R, Friedman R, et al: Expression of Id-1 is regulated by MCAM/MUC18: A missing link in melanoma progression. Cancer Res 71: 3494-3504, 2011.

(i) $\ominus$ This work is licensed under a Creative Commons Attribution-NonCommercial-NoDerivatives 4.0 International (CC BY-NC-ND 4.0) License. 